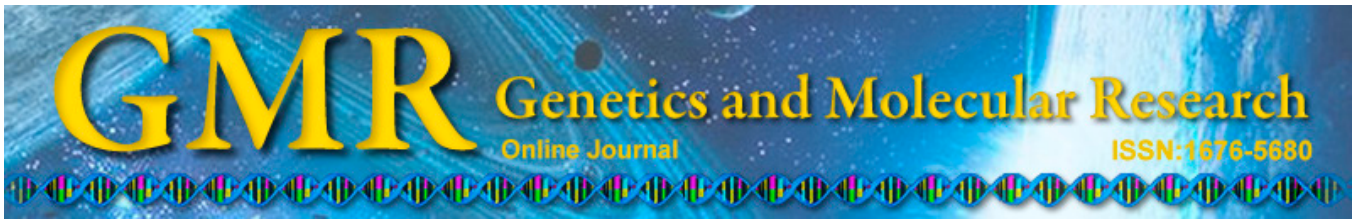

\title{
Comparison of genetic diversity between wild-caught broodstock and hatchery-produced offspring populations of the vulnerable Korean kelp grouper (Epinephelus bruneus) by microsatellites
}

\author{
H.S. An ${ }^{1}$, S.G. Yang 2 , T.S. Moon ${ }^{2}$, J.Y. Park ${ }^{3}$, C.G. Hong ${ }^{3}$, H.K. Hwang ${ }^{4}$, \\ J.I. Myeong ${ }^{4}$ and C.M. An ${ }^{1}$ \\ ${ }^{1}$ Biotechnology Research Division, \\ National Fisheries Research and Development Institute, Busan, Korea \\ ${ }^{2}$ Future Aquaculture Research Center, Jeju-do, Korea \\ ${ }^{3}$ Aquaculture Industry Division, \\ National Fisheries Research and Development Institute, Yeosu, Korea \\ ${ }^{4}$ Aquaculture Management Division, \\ National Fisheries Research and Development Institute, Busan, Korea \\ Corresponding author: H.S. An \\ E-mail: hsan97@korea.kr
}

Genet. Mol. Res. 13 (4): 9675-9686 (2014)

Received June 13, 2014

Accepted October 31, 2014

Published November 14, 2014

DOI http://dx.doi.org/10.4238/2014.November.14.13

\begin{abstract}
The kelp grouper Epinephelus bruneus (Perciformes: Haemulidae), is one of the most economically important fishery resources in Korea. This fish is regarded as a target for prospective aquaculture diversification; therefore, maintenance of stock quality is important. To investigate the effects of current artificial reproduction in a hatchery facility, genetic variation in wild-caught broodstock and hatchery-produced offspring of kelp grouper was analyzed using eight polymorphic nuclear microsatellite DNA loci; 77 alleles were
\end{abstract}


identified. Allelic variability ranged from 2 to 22 in the broodstock and from 1 to 10 in the offspring. The average observed and expected heterozygosities were 0.620 and 0.623 in the broodstock and 0.600 and 0.513 in the offspring, respectively. The possibility of a recent genetic bottleneck was suggested in both populations of E. bruneus. The minor, but significant, genetic differentiation $\left(F_{\mathrm{ST}}=0.047, \mathrm{P}<0.05\right)$ observed was mainly due to statistically significant reductions in the number of alleles in the offspring compared with the broodstock, suggesting that these genetic changes could be related to genetic drift. Our results demonstrate the usefulness of microsatellite markers to monitor genetic variation and raise concerns about potential harmful genetic effects of inappropriate hatchery procedures. Therefore, genetic variation between broodstock and offspring in a hatchery should be monitored in both breeding and release programs as a routine hatchery operation, and inbreeding should ideally be controlled to improve kelp grouper hatchery management. Our data provide a useful genetic basis for future planning of sustainable culture and management of E. bruneus in fisheries.

Key words: Korean kelp grouper; Longtooth grouper; Genetic variability; Microsatellite loci; Artificial reproduction

\section{INTRODUCTION}

Epinephelus bruneus, commonly known as kelp grouper, is of considerable importance in commercial fisheries. This is a bottom-associated fish that inhabits rocky reefs and muddy bottoms, and is narrowly distributed in tropical and subtropical seas, including the seas of Korea, Japan, China, Hong Kong, and Taiwan (Heemstra and Randall, 1993). Based on a decline in catch data in its relatively narrow range, E. bruneus is listed as an endangered species in Earth's Endangered Creatures (EEC, www.earthsendangered.com) and as a vulnerable species on the International Union for the Conservation of Nature and Natural Resources (IUCN) Red List of Threatened Species (IUCN, 2014). Bottom trawling within its habitat is intensive, but there is little fisheries management and fishing pressure appears to be increasing with declines inferred in other parts of its range (Thierry et al., 2008).

In Korea, E. bruneus is an important and expensive marine fish species. Its high commercial value, robustness, and rapid growth in overcrowded conditions have made E. bruneus a good candidate species for aquaculture diversification. Complete culture for sustainable fry production of kelp grouper, including reproduction control, captive spawning, hatching, and larval and juvenile rearing, became possible in the early 2000s. However, aquaculture systems are not well controlled and are still small-scale operations, which are often insufficient in terms of both quantity and quality, making it difficult to meet demand. In addition, to enhance fishery harvests, the Korean government has recently commenced a resource enhancement program involving the release of hatchery-reared fish into natural coastal areas (Korean Fisheries Resources Agency, 2012).

Typically, almost all kelp grouper hatcheries in Korea maintain wild-caught sources of E. bruneus as broodstock for artificial reproduction. The fish is a protogynous hermaphrodite, 
beginning life as female before becoming male. Milt collection is difficult, as the large size of mature male broodfish limits their numbers (Kime et al., 2001). In practice, the offspring of kelp groupers usually breed in an uncontrolled manner. Thus, there is concern that genetic variability among hatchery stocks may be lost during artificial propagation. Loss of genetic variation is potentially harmful, compromising various commercially important traits, including survival and growth, which can render aquaculture uneconomical (Allendorf and Ryman, 1987). The combination of intensive stocking practices with inappropriate hatchery procedures may have detrimental genetic impacts on wild stock, accompanied by the risk that continued exploitation of the resource may become unviable (Kohlmann et al., 2005; Wang et al., 2011; An et al., 2013). Information on standard breeding practices in hatcheries is needed to ensure that cultivated stock is managed in a manner that allows conservation of diversity and minimization of inbreeding, which would ensure the future viability of aquaculture programs. Therefore, genetic diversity should ideally be routinely monitored during both breeding and release to improve hatchery management.

Molecular genetic diversity in fish has been found to be associated with life history traits, which reflect habitat types (DeWoody and Avise, 2000). Therefore, it is necessary to investigate genetic variability in these wild-caught broodstock and hatchery-produced offspring populations of kelp grouper to obtain well-founded scientific data fundamental to the success of aquaculture development strategies. Molecular markers have proven to be an exceptional indicator of genetic variation within and between populations of many fishery animals (Choi and Kim, 2012; Hong et al., 2012; Lee and Hur, 2012). Among the available genetic markers, microsatellite DNA markers or short tandem repeats (STRs) are well-known hypervariable genetic markers that have been successfully used in genetic monitoring of the changes between wild and hatchery populations in many marine species (An et al., 2012b; Han et al., 2012; Kim et al., 2013). Recently, microsatellite DNA markers for E. bruneus have been isolated and characterized (An et al., 2012a; Kang et al., 2013); however, there have been no reports regarding their use in analysis of the extent of genetic divergence between wild-caught broodstock and hatchery-produced offspring of Korean kelp grouper in hatcheries, although great efforts are devoted to sustainable mass fry production.

The principal objective of this preliminary study was to genetically characterize the broodstock and offspring populations of a hatchery facility located on Jeju Island, Korea, to determine whether the current management strategy reduces genetic variation in E. bruneus. Such data are necessary to establish founding principles and to maintain cultivated stock in a manner that conserves diversity and minimizes inbreeding.

\section{MATERIAL AND METHODS}

\section{Sample collection and DNA extraction}

Fin-clip tissue samples $\left(\sim 1 \mathrm{~cm}^{3}\right)$ were obtained from a hatchery located on Jeju Island, Korea, in which offspring of kelp grouper are reared every year. At this facility, approximately 70 wild-caught breeders over 5 years of age are maintained for reproduction. In 2013, we sampled 50 fish selected for reproduction on the basis of sexual maturity and good health. After mating, offspring were reared in a tank. One year after hatching, 50 offspring were randomly sampled. All samples were stored in $2 \mathrm{~mL}$ of $99 \%(\mathrm{v} / \mathrm{v})$ ethanol at $4{ }^{\circ} \mathrm{C}$ prior to DNA extraction. Total genomic DNA was extracted from fin-clips using an automated DNA extrac- 
tion system (MagExtractor MFX-2100; Toyobo, Osaka, Japan) and a MagExtractor-Genomic DNA Purification Kit (Toyobo). The DNA extracted was stored at $-20^{\circ} \mathrm{C}$ prior to genotyping.

\section{Microsatellite genotyping}

Nine previously characterized highly variable microsatellite loci were selected for analysis (Kang et al., 2013). One-hundred kelp groupers from two populations (wild-caught broodstock and hatchery-produced offspring) were typed. Primer sequences, microsatellite repeat sequences, and the optimal annealing temperatures for each locus are listed in Table 1.

The forward primer from each primer set was fluorescently labeled at the 5'-end with one of three dyes: 6-FAM, HEX, or NED (PE Applied Biosystems, Foster City, CA, USA). The nine microsatellite loci were amplified by PCR using an RTC 200 instrument (MJ Research, Watertown, MA, USA) in $10 \mu \mathrm{L}$ reaction mixtures containing 10-50 ng DNA, $1 \mathrm{X}$ ExTaq buffer, $0.2 \mathrm{mM}$ dNTPs, 10 pmol each primer, and $0.25 \mathrm{U}$ Taq DNA polymerase (Takara, Shiga, Japan). The thermal cycling protocol consisted of initial denaturation for $11 \mathrm{~min}$ at $95^{\circ} \mathrm{C}$, followed by 35 cycles of $1 \mathrm{~min}$ at $94^{\circ} \mathrm{C}, 1 \mathrm{~min}$ at $60^{\circ} \mathrm{C}$, and $1 \mathrm{~min}$ at $72^{\circ} \mathrm{C}$, with a final 5 min extension at $72^{\circ} \mathrm{C}$. For genotyping, $1 \mu \mathrm{L}$ aliquots of PCR products were added to formamide-containing reaction mixtures containing a size standard [GeneScan-400HD (ROX); PE Applied Biosystems] and electrophoresed on an ABI3130 DNA sequencer (PE Applied Biosystems). Fragment length was determined using the GeneMapper software (v4.0; PE Applied Biosystems).

Table 1. Nine microsatellite loci sequences of kelp grouper (Epinephelus bruneus) examined in this study, with core repeats. The primers shown were used for PCR amplification.

\begin{tabular}{|c|c|c|c|c|}
\hline Locus & Primer sequence $\left(5^{\prime}-3^{\prime}\right)$ & Repeats & $\mathrm{Ta}\left({ }^{\circ} \mathrm{C}\right)$ & Reference \\
\hline $\mathrm{Eb} 1$ & $\begin{array}{l}\text { F: GAGGGAAGACAGCCCTAGTAGT ned } \\
\text { R: AAACGATTTGATTACGGAATTG }\end{array}$ & $(\mathrm{TG})_{10}$ & 60 & \\
\hline $\mathrm{Eb} 6$ & $\begin{array}{l}\text { F: GTTTGACTCACTCACTCCCTTC hex } \\
\text { R: GTAAGAGCGTACTGAGCTGGTT }\end{array}$ & $(\mathrm{CA})_{14}$ & 60 & \\
\hline $\mathrm{Eb} 8$ & $\begin{array}{l}\text { F: ATCTTGTGGAGCCTTTTAGTGA ned } \\
\text { R: CAGGAAATAAGACAGGAAGCAC }\end{array}$ & $(\mathrm{TG})_{17}$ & 60 & \\
\hline $\mathrm{Eb} 22$ & $\begin{array}{l}\text { F: AAGCAAAAAGCTTTGAGAGTTG hex } \\
\text { R: ATACACACGTGGACAGCAGAC }\end{array}$ & $(\mathrm{TG})_{12}$ & 60 & \\
\hline $\mathrm{Eb} 28$ & $\begin{array}{l}\text { F: CATAGCAGTGGTTGAGAAACAA ned } \\
\text { R: CTTCAAACATGGATCAAGACAA }\end{array}$ & $(\mathrm{CA})_{12}$ & 60 & Kang et al. (2013) \\
\hline $\mathrm{Eb} 45$ & $\begin{array}{l}\text { F: ATCTACTCTGTCGGCACAAGAT hex } \\
\text { R: CATAGTCCACCTGAAAGGTCAT }\end{array}$ & $(\mathrm{TG})_{13}$ & 60 & \\
\hline $\mathrm{Eb} 57$ & $\begin{array}{l}\text { F: CAACAGTGTGAACCAAAACAAT 6-fam } \\
\text { R: ACCATGTCTTTGTTGCTGCT }\end{array}$ & $(\mathrm{CA})_{19}$ & 60 & \\
\hline $\mathrm{Eb} 60$ & $\begin{array}{l}\text { F: TGTCAAATTATCAACAAGCCAG 6-fam } \\
\text { R: TATGTTGACTTTGGGAAGCTCT }\end{array}$ & $(\mathrm{CA})_{15}$ & 60 & \\
\hline $\mathrm{Eb} 64$ & $\begin{array}{l}\text { F: ATGTTCACTTTTCTCGTCCTGT 6-fam } \\
\text { R: CTGTCAGGTTCGTAGACAGAAA }\end{array}$ & $(\mathrm{TG})_{13}$ & 60 & \\
\hline
\end{tabular}

$\mathrm{Ta}=$ optimal annealing temperature.

\section{Population comparison}

Individual genotypes were scored using the GeneMapper (v4.0; Applied Biosystems) with a size standard and an internal control for allele calling; each allele was coded according to its size in nucleotide base pairs (bp). A panel including all alleles detected in the 100 individuals was created for each locus. Possible null alleles and genotyping errors caused by 
stuttering and/or large-allele dropout were evaluated using MICRO-CHECKER (1000 randomizations; van Oosterhout et al., 2004). Scoring errors and human errors were estimated via duplicate analysis.

The genetic diversity of each sample was calculated using the number of alleles $\left(N_{\mathrm{A}}\right)$, the size range of each allele (in bp; S), the number of unique alleles observed $(U)$, the observed heterozygosity $\left(H_{\mathrm{O}}\right)$, the expected heterozygosity $\left(H_{\mathrm{E}}\right)$, and the PIC calculated using the CERVUS v3.03 (Kalinowski et al., 2007). Differences in genetic diversity parameters were identified using nonparametric analysis (Wilcoxon signed-rank test; Wilcoxon, 1945). Deviations from linkage disequilibrium (a measure of the extent of distortion from independent segregation of loci) were evaluated using the ARLEQUIN v3.0 (Excoffier et al., 2005) by a permutation procedure (Slatkin and Excoffier, 1996). Deviations from Hardy-Weinberg equilibrium (HWE) were evaluated using the GENEPOP'007 (Rousset, 2008) employing inbreeding coefficients $\left(F_{\text {IS }}\right.$ values; Weir and Cockerham, 1984). Significance levels were adjusted to reflect the fact that multiple tests had been run using sequential Bonferroni correction (Rice, 1989). The Bottleneck software program v1.2.02 (Cornuet and Luikart, 1996) was used under the infinite allele model (IAM), stepwise-mutation model (SMM), and two-phase model of mutation (TPM) with 1000 iterations to verify the existence of bottlenecks inferred by heterozygosity excess in the populations. Significance was tested using the Wilcoxon signed-rank test (Wilcoxon, 1945).

The extent of between-population differences was examined by calculating the global multilocus $F_{\mathrm{ST}}$ (Weir and Cockerham, 1984) and $R_{\mathrm{ST}}$ values (1000 permutations; Rousset, 1996). The indices of pairwise $F_{\mathrm{ST}}$ values obtained using an IAM and the $R_{\mathrm{ST}}$ values calculated using a SMM were determined using the ARLEQUIN v3.0. The log-likelihood G test (Goudet et al., 1996) within GENEPOP'007 was used to determine whether the allelic and genotypic distributions were identical between the two populations. The significances of $\mathrm{P}$ values across all loci for the two populations were determined using Fisher's probability combination test and evaluated following the sequential Bonferroni adjustment of critical probabilities (Rice, 1989). For analysis of molecular variance (AMOVA; Excoffier et al., 1992), components of variance both within and between populations (based on IAM data) were estimated using the ARLEQUIN v3.0. The significance of identified AMOVA components was tested by running 1000 permutations.

\section{RESULTS}

\section{Genetic diversity}

Two populations of wild-caught broodstock and hatchery-produced offspring of $E$. bruneus (total $\mathrm{N}=100$ ) were screened for genetic variations at nine polymorphic microsatellite loci. The nine primer sets yielded variable profiles and reruns were performed on 16 individuals of each sample set to ensure that allelic scoring was reproducible. No significant differences were observed when original and rerun data were compared, indicating that genotyping errors did not affect allelic scoring. Individual genotypes that were not scorable after the first PCR amplification were identified after only one additional PCR run, reducing the likelihood that our results were affected by poor quality DNA.

The MICRO-CHECKER analysis suggested that two of the nine loci (loci Eb 1 and Eb 45) may include null alleles, and our data demonstrated that Eb 45 was affected by one 
or more null alleles in both populations. To minimize the detection of false positives, we considered this one locus (Eb 45) to have null alleles, and it was therefore eliminated from subsequent analyses. We estimated global multilocus $F_{\mathrm{ST}}$ with and without this locus. None of the other eight loci exhibited highly significant linkage disequilibrium $(\mathrm{P}>0.05)$.

All nine microsatellite loci were polymorphic in the two populations of kelp grouper, and the level of polymorphism varied among loci. Specifically, Eb 28 showed both greater allelic diversity and higher levels of heterozygosity. The measures of genetic diversity for each population calculated from the observed allele distributions are shown in Table 2. DNA amplification of the 100 samples generated a total of 77 alleles over the eight loci, ranging from two alleles at locus $\mathrm{Eb} 8$ to 23 at locus $\mathrm{Eb} 28$, with an average of 9.63 alleles per locus. Neither population exhibited a diagnostic allele. A small-to-high extent of polymorphism was detected at each locus; the PIC values ranged between 0.000 and 0.937 (Table 2). The mean observed and expected heterozygosities ranged from 0.240 and 0.292 at locus Eb 1 to 0.990 and 0.889 at Eb 28, respectively (Table 2).

Differences in genetic diversity were also evident between wild-caught broodstock

Table 2. Summary statistics for eight microsatellite loci in the broodstock and offspring populations of kelp grouper (Epinephelus bruneus).

\begin{tabular}{|c|c|c|c|c|c|c|c|c|c|c|}
\hline \multirow[t]{2}{*}{ Population (N) } & & \multicolumn{8}{|c|}{ Microsatellite loci } & \multirow[b]{2}{*}{ Mean } \\
\hline & & $\mathrm{Eb} 1$ & $\mathrm{~Eb} 6$ & $\mathrm{~Eb} 8$ & $\mathrm{~Eb} 22$ & $\mathrm{~Eb} 28$ & $\mathrm{~Eb} 57$ & $\mathrm{~Eb} 60$ & $\mathrm{~Eb} 64$ & \\
\hline \multirow{4}{*}{ Broodstock (50) } & $F_{\mathrm{ST}}$ & 0.039 & 0.033 & 0.047 & 0.088 & 0.077 & 0.021 & 0.069 & 0.004 & 0.047 \\
\hline & $N_{\mathrm{A}}^{\mathrm{s}}$ & 5 & 11 & 2 & 7 & 22 & 8 & 15 & 5 & 9.38 \\
\hline & $S^{\mathrm{A}}$ & $258-266$ & $175-205$ & $237-243$ & $194-212$ & $231-281$ & $132-156$ & $138-172$ & $144-154$ & \\
\hline & $U$ & 3 & 8 & 1 & 2 & 13 & 3 & 9 & 2 & 5.125 \\
\hline \multirow{9}{*}{ Offspring (50) } & $H_{\mathrm{E}}$ & 0.401 & 0.694 & 0.096 & 0.802 & 0.950 & 0.618 & 0.881 & 0.539 & 0.623 \\
\hline & $H_{\mathrm{O}}$ & 0.280 & 0.660 & 0.100 & 0.820 & 1.000 & 0.560 & 0.860 & 0.680 & 0.620 \\
\hline & PIC & 0.368 & 0.658 & 0.090 & 0.763 & 0.937 & 0.550 & 0.861 & 0.437 & 0.583 \\
\hline & $F_{\text {IS }}$ & $0.305^{*}$ & 0.049 & -0.043 & -0.023 & -0.054 & 0.094 & 0.024 & $-0.265^{*}$ & 0.011 \\
\hline & $N_{\mathrm{A}}^{\mathrm{IS}}$ & 2 & 3 & 1 & 6 & 10 & 5 & 6 & 3 & 4.50 \\
\hline & $S^{\mathrm{A}}$ & $258-262$ & $175-195$ & 243 & $194-214$ & $245-283$ & $132-152$ & $138-158$ & $144-150$ & \\
\hline & $U$ & 0 & 0 & 0 & 1 & 1 & 0 & 0 & 0 & 0.250 \\
\hline & $H_{\mathrm{E}}$ & 0.182 & 0.483 & 0.000 & 0.775 & 0.828 & 0.594 & 0.713 & 0.528 & 0.513 \\
\hline & $H_{\mathrm{O}}^{\mathrm{E}}$ & 0.200 & 0.520 & 0.000 & 0.980 & 0.980 & 0.680 & 0.820 & 0.620 & 0.600 \\
\hline \multirow{5}{*}{ Mean all population } & PIC & 0.164 & 0.402 & 0.000 & 0.727 & 0.797 & 0.501 & 0.663 & 0.414 & 0.459 \\
\hline & $F_{\mathrm{IS}}$ & -0.101 & -0.078 & 0.000 & $-0.268 *$ & $-0.185^{*}$ & -0.146 & -0.151 & -0.177 & -0.138 \\
\hline & $N^{\text {IS }}$ & 3.5 & 7 & 1.5 & 6.5 & 16 & 6.5 & 10.5 & 4 & 6.938 \\
\hline & $H_{\mathrm{E}}^{\mathrm{A}}$ & 0.292 & 0.589 & 0.048 & 0.789 & 0.889 & 0.606 & 0.797 & 0.534 & 0.568 \\
\hline & $H_{\mathrm{O}}^{\mathrm{E}}$ & 0.240 & 0.590 & 0.050 & 0.885 & 0.990 & 0.620 & 0.840 & 0.650 & 0.608 \\
\hline
\end{tabular}

Single-locus $F_{\mathrm{ST}}, \mathrm{N}=$ number of samples, $N_{\mathrm{A}}=$ number of alleles per locus, $S=$ size in bp of alleles, $U=$ number of unique alleles, $H_{\mathrm{E}}=$ expected heterozygosity, $H_{\mathrm{O}}=$ observed heterozygosity, $\mathrm{PIC}=$ polymorphism information content and $F_{\text {IS }}=$ inbreeding coefficient are given for each population and locus. Calculations assume that individuals with one microsatellite band are homozygous for the allele. *Significant deviation from HardyWeinberg equilibrium after Bonferroni correction (P, initial $\alpha=0.05 / 8=0.006)$.

and their hatchery-produced offspring. The allelic frequencies at all eight selected loci of each population are shown in Figure 1; between-population differences are evident. Significantly greater numbers of alleles were found in wild-caught broodstock compared with hatcheryproduced offspring (75 vs. 36, respectively; average allele No. $9.38 v s .4 .50$, respectively; Wilcoxon signed-rank test, $\mathrm{P}<0.05$ ). In total, 43 alleles were unique to single populations (Table 2), but the offspring had only two unique alleles (i.e., 41 in broodstock $v s .2$ in offspring).

The average expected heterozygosities were 0.623 and 0.513 in broodstock and off- 
spring, respectively. The corresponding estimates were 0.620 and 0.600 , respectively (Table $2)$. There were no significant differences between broodstock and offspring in terms of heterozygosity. Inbreeding coefficients ( $F_{\text {IS }}$ values) varied among markers, from -0.265 (Eb 64) to 0.305 (Eb 1) in broodstock and from -0.268 (Eb 22) to 0.000 (Eb 8) in offspring. Average $F_{\text {IS }}$ values, including those of all markers, were 0.011 in broodstock and -0.138 in offspring.

Significant departures from HWE after Bonferroni correction $(\mathrm{P}<0.006)$ were observed in two loci (Eb 1 and $\mathrm{Eb} 64)$ in the wild-caught broodstock population and two loci (Eb 22 and $\mathrm{Eb} 28)$ in the hatchery-produced offspring population, indicating that deviations from HWE were mainly attributable to heterozygote excess, except at one locus (Eb 1).

The allelic frequencies of all eight microsatellites of each sample are shown in Figure 1. The frequency distributions indicate the presence of 36 rare alleles (frequency $<5 \%$ ) of a total of 75 alleles over all loci (mean 48\%) in the wild-caught broodstock and 10 rare alleles of a total of 36 alleles (mean 27.8\%) in the hatchery-produced offspring. Rare alleles were evident at most loci and were therefore not associated with any particular locus in either population.

The mutation drift equilibrium tests for the detection of genetic bottlenecks showed that both populations exhibit heterozygosity excess of microsatellites under the IAM (Wilcoxon test, $\mathrm{P}<0.05$ ). These results likely indicate a bottleneck in these populations.

\section{Genetic variation between samples}
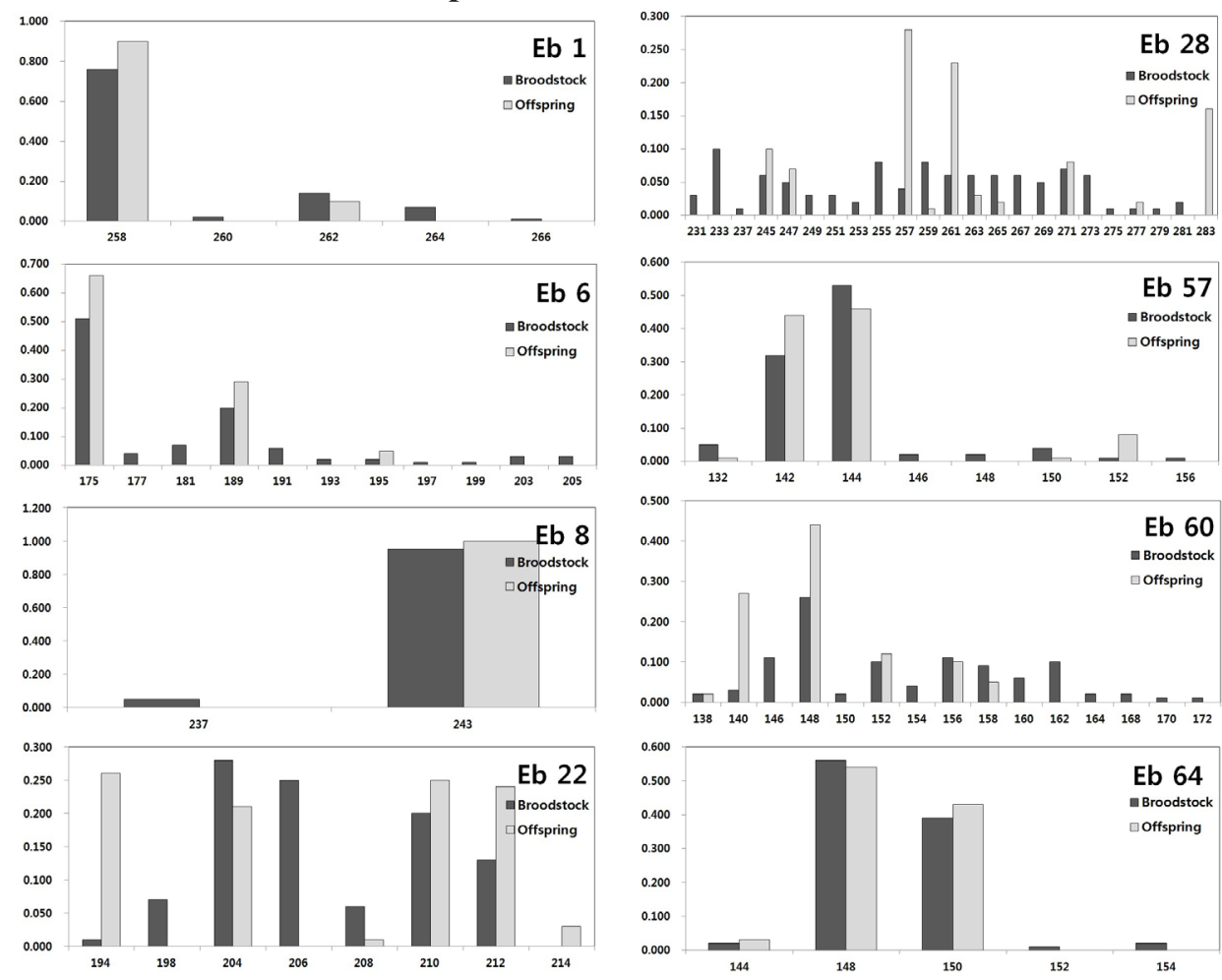

Figure 1. Allele frequency (y-axis) and allele base-pair length (x-axis) distributions of the eight microsatellite loci in the broodstock and offspring populations of Epinephelus bruneus used in this study. 
Genetic variation between the wild-caught broodstock and the hatchery-produced offspring was estimated by calculating $F_{\mathrm{ST}}$ and $R_{\mathrm{ST}}$ values. The global multilocus $F_{\mathrm{ST}}$, including the values of all loci, was $0.047(\mathrm{P}<0.001)$ and the $R_{\mathrm{ST}}$ value was $0.083(\mathrm{P}<0.001)$. When Eb 45 was included, the global multilocus $F_{\mathrm{ST}}$ was $0.057(\mathrm{P}<0.001) . F_{\mathrm{ST}}$ estimates differed significantly between broodstock and offspring irrespective of whether the single locus exhibiting significant departures from HWE in both populations (Eb 45) was included. These results indicate a significantly low level of genetic variation between the two populations. AMOVA of all eight microsatellites yielded similar results to those of the FSTAT analysis in terms of variation: $96.6 \%(\mathrm{P}=0.231)$ within individuals, $-1.33 \%(\mathrm{P}=0.773)$ among individuals within populations, and $4.73 \%(\mathrm{P}=0.000)$ among populations. AMOVA indicated that genetic variation was significant between the two sample sets, although the low level of such variation was explained at the "among populations" hierarchical level (4.73\%). Thus, low-level variation between broodstock and offspring was supported by AMOVA (Table 3).

Genetic variation between the two populations was also observed via pairwise comparisons of allelic and genotypic frequencies. Such comparisons indicated that of the 16 locuspopulation pairs examined, 11 differed significantly between the two populations in both allelic and genotypic frequencies after sequential Bonferroni correction (Table 4).

$\begin{aligned} & \text { Table 3. Analysis of molecular variance (AMOVA) of eight microsatellite loci in the broodstock and offspring } \\
& \text { populations of Epinephelus bruneus. }\end{aligned}$
\begin{tabular}{lrcccc}
\hline Source of variation & d.f. & Sum of squares & Variance components & Percentage variation (\%) & P value \\
\hline Among populations & 1 & 18.315 & 0.15758 & 4.73 & 0.00000 \\
Among individuals & 98 & 250.570 & -0.03658 & -1.33 & 0.77322 \\
Within population & 100 & 263.000 & 2.63000 & 96.60 & 0.23069 \\
Within individuals & 199 & 531.885 & 2.75100 & & \\
Total &
\end{tabular}

Table 4. Comparison of allelic and genotypic frequencies at eight microsatellite loci between the broodstock and offspring populations of Epinephelus bruneus.

\begin{tabular}{lccc}
\hline Locus & P value (allelic frequencies) & Locus & P value (genotypic frequencies) \\
\hline Eb 1 & $0.002^{*}$ & Eb 1 & 0.024 \\
Eb 6 & $0.000^{*}$ & Eb 6 & $0.000^{*}$ \\
Eb 8 & 0.058 & Eb 8 & 0.055 \\
Eb 22 & $0.000^{*}$ & Eb 22 & $0.000^{*}$ \\
Eb 28 & $0.000^{*}$ & Eb 28 & $0.000^{*}$ \\
Eb 57 & $0.006^{*}$ & Eb 57 & $0.005^{*}$ \\
Eb 60 & $0.000^{*}$ & Eb 60 & $0.000^{*}$ \\
Eb 64 & Eb 64 & 0.501 \\
\hline
\end{tabular}

Probability values of homogeneity of allelic frequency distributions (P) estimated by a test analogous to the Fisher exact test in the Markov-chain method are shown; wide significance levels were applied using the sequential Bonferroni technique $(k=8)$. Significant at $\mathrm{P}<0.006$.

\section{DISCUSSION}

In the present study, the genetic variation in wild-caught broodstock samples of E. bruneus (mean $N_{\mathrm{A}}=9.38$ and mean $H_{\mathrm{E}}=0.62$ ) was lower than that reported (mean 
$N_{\mathrm{A}}=19.96 \pm 6.6$, mean $H_{\mathrm{E}}=0.77 \pm 0.19$; averages of 12 species) for other marine fish (DeWoody and Avise, 2000). Similar genetic variability has been reported for other demersal fish species, such as the Korean wild starry flounder (Platichthys stellatus; An et al., 2011a) and the Korean black rockfish (Sebastes inermis; An et al., 2011b), and for other serranid species (Wang et al., 2011; An et al., 2012b, 2014), suggesting that demersal serranid fish species may be less diverse, small, and isolated compared to migratory fish.

When the level of diversity in the hatchery-produced offspring population was compared with that of the wild-caught broodstock population, no significant difference was observed in the average expected heterozygosity but there was a significant difference in the average number of alleles per locus between the two populations; i.e., on average, $52 \%$ reduction in the number of alleles, $95.1 \%$ reduction in the number of unique alleles, and $17.7 \%$ reduction in expected heterozygosity were observed in offspring. Many studies have found that reductions in allelic diversity and/or mean numbers of alleles per locus can occur in the absence of marked changes in heterozygosity, when hatchery and wild populations were compared (Reily et al., 1999; An et al., 2011a,b). The loss of alleles is more important than a change in allelic frequency because the latter may change again by random drift but a lost allele cannot be recovered. As a high degree of genetic variation is considered to be associated with adaptive fitness in terms of changing environments, loss of genetic variation is detrimental to domestication of cultured stocks (Koljonen et al., 2002). Thus, genetic factors are of vital importance to ensure production of high-quality marine animal seed. In the present study, the decline of genetic variation in offspring may have been caused principally by founder effects developing in breeding programs that use small broodstock numbers, triggering a loss of genetic diversity in offspring. The use of only a few wild individuals as broodstock may greatly affect genetic diversity, especially allelic diversity (Allendorf and Ryman, 1987). Most hatcheries breeding kelp grouper are small or medium in size and have inadequate numbers of parental fish, mainly because wild spawners, the major source of hatchery broodstock, are difficult to catch. The decline in genetic variation of offspring may be due to an increase in genetic drift resulting in turn from the use of small numbers of breeders. Therefore, progeny production should be based on well-managed broodstock strategies. Protection of the genetic characteristics of cultured stock should be taken into consideration in planning artificial reproduction.

Significant deviations from HWE were observed in the wild-caught broodstock and hatchery-produced offspring populations examined. These departures resulted from an excess of heterozygosity in both populations, and a deficit of heterozygosity was mainly detected in the wild-caught broodstock population. In the wild population, heterozygote deficit can be explained by several factors, such as the presence of unrecognized null alleles, natural selection acting on genetic markers, mating among relatives, the reduction of heterozygosity in a population caused by a subpopulation structure known as the Wahlund's effect, or a combination of these factors. In the present case, MICRO-CHECKER analysis revealed the presence of a null allele at the microsatellite locus, Eb 1, which may explain the observed deviation in the wildcaught broodstock population. The importance of null alleles for heterozygote deficiency has been discussed previously for marine fish (Callen et al., 1993; De Sousa et al., 2005). In this study, three of the four significant HWE deviations in the two populations revealed heterozygote excess. Heterozygote excess in a population is not as common as heterozygote deficiency, and therefore has not been fully theoretically explored. Korean kelp grouper populations have declined continuously. This reduction would also have resulted in a corresponding reduction 
in effective population size, and a decrease in effective population size can give rise to a reduction in homozygotes because the sampling bias of alleles can result from a small number of parents and differences in allele frequencies between the sexes (Fujio et al., 1985; Spencer et al., 2000). In addition, it has been reported that a reduction in broodstock population size in a hatchery could increase the heterozygote excess in the progeny due to binominal sampling error of alleles (Luikart and Cournuet, 1999). Hence, a decrease in effective population size and the founder effect may have contributed to the heterozygote excess observed in this study.

Significant genetic differentiation (detected by calculations of $F_{\mathrm{ST}}$ and $R_{\mathrm{ST}}$ ) was evident between broodstock and offspring in the present study. Such differences, particularly in the numbers of unique alleles, are probably related to a reduction in the effective numbers of contributing parents and the effects of artificial selection on hatchery progeny. Hence, genetic drift has probably played an important role in the observed loss of genetic diversity, causing offspring to differentiate from broodstock. As our study was limited by the small number of populations screened, the genetic diversity parameters of either population may be explained if further data on additional populations were available. Although E. bruneus is in an early stage of domestication, both the sample size and sampling locations should be expanded. Further studies are required to assess the influence of standard hatchery breeding practices on the viability of this important fishery species.

Maintenance of genetic variation comparable to that of wild populations within hatchery stocks requires the institution of good broodstock management practices. Parental similarity is negatively related to adult reproductive success, and whenever sample sizes are large, such relationships become significantly negative (Amos et al., 2001). In addition, even if the founder broodstock is genetically intact, lack of a proper management strategy can lead to inbreeding and a rapid decline in genetic diversity. Hence, continued genetic monitoring of bloodstock is warranted if seed is to be artificially produced. In addition, to ensure proper management of stock enhancement programs, progeny production should be founded on wellorganized broodstock management strategies. Genetic factors are of vital importance in the production of good quality marine animal seed for return to the sea. The genetic integrity of wild populations should be protected from the impact of hatchery releases by carefully planning broodstock management strategies.

In summary, genetic diversity analyses revealed substantial changes in genetic variation and significant genetic differentiation between wild-caught broodstock and hatchery-produced offspring populations of kelp grouper in a Korean hatchery. These results suggest that genetic drift may exert negative effects on the reproductive capacity of the stock, because genetic factors are important in the production of high quality seed. Our data are important to ensure the success of ongoing breeding and stock enhancement programs. Thus, careful broodstock management will be necessary to ensure the success of artificial seed production. The data presented here will provide a useful genetic basis for the planning of future sustainable fry production and for management of E. bruneus in fisheries.

\section{ACKNOWLEDGMENTS}

Research supported by the National Fisheries Research and Development Institute (NFRDI; contribution \#RP-2014-BT-023). The views expressed herein are those of the authors and do not necessarily reflect the views of NFRDI. 


\section{REFERENCES}

Allendorf FW and Ryman N (1987). Genetic management of hatchery stocks. In: Population genetics and fishery management (Ryman N and Utter F, eds). University of Washington Press, Seattle, WA, pp. 141-159.

Amos W, Wilmer JW, Fullard K, Burg TM, et al. (2001). The influence of parental relatedness on reproductive success. Proc. R. Soc. B-Biol. Sci. 268: 20212027.

An HS, Byun SG, Kim YC, Lee JW, et al. (2011a). Wild and hatchery populations of Korean starry flounder (Platichthys stellatus) compared using microsatellite DNA marker. Int. J. Mol. Sci. 12: 9189-9202.

An HS, Kim EM, Lee JH, Noh JK, et al. (2011b). Population genetic structure of wild and hatchery black rockfish Sebastes inermis in Korea, assessed using cross-species microsatellite markers. Genet. Mol. Res. 10: 2492-2504.

An HS, Kim JW, Lee JW, Kim SK, et al. (2012a). Development and characterization of microsatellite markers for an endangered species, Epinephelus bruneus, to establish a conservation program. Anim. Cells Syst. 16: 50-56.

An HS, Lee JW and Dong CM (2012b). Population genetic structure of Korean pen shell (Atrina pectinata) in Korea inferred from microsatellite marker analysis. Genes Genom. 34: 681-688.

An HS, Lee JW, Park JY and Jung HT (2013). Genetic structure of the Korean black scraper Thamnaconus modestus inferred from microsatellite marker analysis. Mol. Biol. Rep. 40: 3445-3456.

An HS, Kang HW, Han HS, Park JY, et al. (2014). Isolation and characterization of 26 novel polynucleotide microsatellites from short barbeled grunter (Hapalogenys nitens) for genetic analysis. Conserv. Genet. Resour. 6: 669-672.

Callen DF, Thompson AD, Shen Y, Phillips HA, et al. (1993). Incidence and origin of "null" alleles in the (AC)n microsatellite markers. Am. J. Hum. Genet. 52: 922-927.

Choi CG and Kim JM (2012). Detection of Laminariaceae species based on PCR by family-specific ITS primers. Fish. Aquat. Sci. 15: 157-162.

Cornuet JM and Luikart G (1996). Description and power analysis of two tests for detecting recent population bottlenecks from allele frequency data. Genetics 144: 2001-2014.

De Sousa SN, Finkeldey R and Gailing O (2005). Experimental verification of microsatellite null alleles in Norway spruce (Picea abies [L.] Karst.): implications for population genetic studies. Plant Mol. Biol. Rep. 23: 113-119.

DeWoody JA and Avise JC (2000). Microsatellite variation in marine, freshwater and anadromous fishes compared with other animals. J. Fish Bio. 56: 461-473.

Excoffier L, Laval G and Schneider S (2005). ARLEQUIN (version 3.0). An integrated software package for population genetics data analysis. Evol. Bioinform. Online. 1: 47-50.

Excoffier L, Smouse PE and Quattro JM (1992). Analysis of molecular variance inferred from metric distances among DNA haplotypes: Application to human mitochondrial DNA restriction data. Genetics 131: 479-491.

Fujio Y, Sasaki N, Sasaki M and Koganezawa A (1985). Genetic aspect of natural and released population of plaice. Bull. Tohoku Reg. Fish. Res. Lab. 47: 51-57 (in Japanese).

Goudet J, Raymond M, de Meeüs T and Rousset F (1996). Testing differentiation in diploid populations. Genetics 144: 1933-1940.

Han HS, Nam BH, Kang JH, Kim YK, et al. (2012). Genetic variation in wild and cultured populations of the sea squirt Halocynthia roretzi inferred from microsatellite DNA analysis. Fish. Aquat. Sci. 15: 151-155.

Heemstra PC and Randall JE (1993). FAO Species Catalogue. Vol. 16. Groupers of the World (Family Serranidae, Subfamily Epinephelinae). FAO Fisheries Synopsis No. 125, Volume 16, Food and Agriculture Organization of the United Nations, Rome.

Hong SE, Kim JG, Yu JN, Kim KY, et al. (2012). Genetic variation in the Asian shore crab Hemigrapsus sanguineus in Korean coastal waters as inferred from mitochondrial DNA sequences. Fish. Aquat. Sci. 15: 49-56.

IUCN Red List of Threatened Species (2014). Version 2014.2. www.iucnredlist.org. Accessed on May15, 2014.

Kalinowski ST, Taper ML and Marshall TC (2007). Revising how the computer program CERVUS accommodates genotyping error increases success in paternity assignment. Mol. Ecol. 16: 1099-1106.

Kang JH, Yang SM, Moon TS, Park JY, et al. (2013). Development of microsatellite markers for the kelp grouper Epinephelus bruneus by 454 pyrosequencing and transfer to related species. Genet. Mol. Res. 12: 5485-5493.

Kim WJ, Shin EH, Kong HJ, Nam BH, et al. (2013). Development of polymorphic microsatellite markers suitable for genetic linkage mapping of olive flounder Paralichthys olivaceus. Fish. Aquat. Sci. 16: 303-309.

Kime DE, Van Look KJ, McAllister BG, Huyskens G, et al. (2001). Computer-assisted sperm analysis (CASA) as a tool for monitoring sperm quality in fish. Comp. Biochem. Physiol. C-Toxicol. Pharmacol. 130: 425-433.

Kohlmann K, Kersten P and Flajshans M (2005). Microsatellite-based genetic variability and differentiation of domesticated, wild and feral common carp (Cyprinus carpio L) populations. Aquaculture 247: 253-266.

Koljonen ML, Tahtinen J, Saisa M, Koskiniemi J (2002). Maintenance of genetic diversity of Atlantic salmon (Salmo 
salar) by captive breeding programmes and the geographic distribution of microsatellite variation. Aquaculture 212: 69-92.

Korean Fisheries Resources Agency (2012). Evaluation of a stock enhancement programme effectiveness. Korean Fisheries Resources Agency, Seoul, Korea. (In Korean).

Lee HJ and Hur SB (2012). Comparison between phylogenetic relationships based on 18S rDNA sequences and growth by salinity of Chlorella-like species (Chlorophyta). Fish. Aquat. Sci. 15:125-135.

Luikart G and Cournuet JM (1999). Estimating the effective number of breeders from heterozygote excess in progeny. Genetics 151: 1211-1216.

Reily A, Elliott NG, Grewe PM, Clabby C, et al. (1999). Genetic differentiation between Tasmanian cultured Atlantic salmon (Salmo salar L.) and their ancestral Canadian population: comparison of microsatellite DNA and allozyme and mitochondrial DNA variation. Aquaculture 173: 459-469.

Rice WR (1989). Analyzing tables of statistical tests. Evolution 43: 223-225.

Rousset F (1996). Equilibrium values of measures of population subdivision for stepwise mutation processes. Genetics 142: $1357-1362$.

Rousset F (2008). Genepop'007: a complete re-implementation of the genepop software for Windows and Linux. Mol. Ecol. Resour. 8: 103-106.

Slatkin M and Excoffier L (1996). Testing for linkage disequilibrium in genotypic data using the Expectation-Maximization algorithm. Heredity 76: 377-383.

Spencer CC, Neigel JE and Leberg PL (2000). Experimental evaluation of the usefulness of microsatellite DNA for detecting demographic bottlenecks. Mol. Ecol. 9: 1517-1528.

Thierry C, Sadovy Y and To AWL (2008). Epinephelus bruneus. In: IUCN 2012. IUCN Red List of Threatened Species. Version 2012.2. Available at www.iucnredlist.org. Accessed May 20, 2014.

van Oosterhout C, Hutchinson WF, Wills DPM and Shipley P (2004). MICRO-CHECKER: Software for identifying and correcting genotyping errors in microsatellite data. Mol. Ecol. Notes. 4: 535-538.

Wang L, Meng Z, Liu X, Zhang Y, et al. (2011). Genetic diversity and differentiation of the orange-spotted grouper (Epinephelus coioides) between and within cultured stocks and wild populations inferred from microsatellite DNA analysis. Int. J. Mol. Sci. 12: 4378-4394.

Weir BS and Cockerham CC (1984). Estimating F-statistics for the analysis of population structure. Evolution 38: 13581370.

Wilcoxon F (1945). Individual comparisons by ranking methods. Biometrics Bull. 1: 80-83. 